\section{PTH-052 A RETROSPECTIVE ANALYSIS OF ENDOSCOPIC MANAGEMENT OF POST LIVER TRANSPLANT BILIARY ANASTOMOTIC STRICTURES}

${ }^{1,2}$ Jonathan Potts*, ${ }^{1}$ Leonardo Eusebi, ${ }^{1}$ Olivia Greenham, ${ }^{1,2}$ Margaret Keane, ${ }^{2}$ George Goodchild, 1,2Kalpesh Besherdas, 1,2Michael Chapman, 1,2 Gavin Johnson, ${ }^{1,2}$ Steve Pereira, 1,2George Webster, ${ }^{1,2}$ Douglas Thorburn. Royal Free Hospital, London, UK ; ${ }^{2}$ University College Hospital, London, UK

\subsection{6/gutjnl-2018-BSGAbstracts.73}

Introduction Biliary anastomotic strictures (AS) occur in $5 \%-15 \%$ of liver transplant (LT) recipients and cause graft dysfunction and morbidity. The main alternative to surgical reconstruction is stenting at endoscopic retrograde cholangiopancreatography (ERCP), using plastic polyethylene or fully-covered self-expanding metal stents (fcSEMS). We retrospectively reviewed outcomes after ERCP for biliary AS, aiming to assess rates of success and complications.

Methods Records for all patients undergoing ERCP post LT between 2013-16 were reviewed. AS were classified as early or late $(<3$ or $\geq 3 \mathrm{mths}$ post LT) and cases of diffuse cholangiopathy excluded. Data collected included graft characteristics, timing/nature of ERCPs and complications. Stricture resolution was determined from graft function, imaging and ERCP findings.

Results In total 252 ERCPs were performed (median 4/ patient, range 2-10) in 62 patients, $72.6 \%$ male, age at LT 54 years (range 20-71). 48 grafts $(77.4 \%)$ were donations after brain death, $13(21.0 \%)$ after cardiac death and 1 live donor. At first endoscopy $53(85.4 \%)$ had a native papilla. Median time from LT was 28 days (4-75) in early AS $(n=29,46.7 \%, 9$ presenting with bile leak) vs 11.5 mths (3.4-251) in late AS $(n=33,53.2 \%)$. Biliary stents were placed in 172 ERCPs $(63.4 \%$ plastic vs $36.6 \%$ fcSEMS) and AS dilated in $68(27.0 \% ; 60$ in conjunction with stenting).

Stricture resolution was achieved endoscopically in 47/55 patients with complete outcome data (85.5\%). Plastic stents were placed at index ERCP in $23 / 26$ of early AS, in whom sequential plastic 'upstenting' was ultimately successful in 9 $(39.1 \%)$ and $12(52.2 \%)$ proceeded to fcSEMS (stricture remodelled in all). Of late AS, 9/29 were managed with fcSEMS (88.8\% successfully), 8 plastic 'upstenting' (75\% success), 8 fcSEMS after initial plastic stent (all successful) and 2 resolved with dilatation only.

The overall rate of post-ERCP pancreatitis (PEP) was 7.1\% (none severe by Cotton criteria) and was higher after fcSEMS $(15.9 \%)$ than plastic stenting $(5.5 \%, \mathrm{p}=0.024)$, despite similar use of NSAID prophylaxis. Compared with plastic stents, fcSEMS were more likely to migrate $(36.5 \%$ vs $12.8 \%$, $\mathrm{p}<0.001)$ and embed $(6.3 \%$ vs $0 \%, \mathrm{p}=0.008)$ but stent occlusion was numerically lower $(0 \%$ vs $4.6 \%, \mathrm{p}=0.084)$. Of 8 endoscopic failures (14.5\%), $3(5.5 \%)$ required percutaneous stenting and $2(3.6 \%)$ biliary reconstruction, one after duodenal perforation by a migrated plastic stent.

Conclusions Endoscopic management is effective in treating $85 \%$ of biliary AS. fcSEMS appear superior to sequential plastic 'upstenting' but are associated with higher rates of PEP and migration.

\section{PTH-053 ENDOCUFF ASSISTED COLONOSCOPY SIGNIFICANTLY IMPROVE ADR COMPARING TO CAP ASSISTED COLONOSCOPY: A RANDOMISED STUDY (DETECT)}

Rajaratnam Rameshshanker*, Zacharias Tsiamoulos, Ana Wilson, Brian Saunders. St Mark's Hospital, Harrow, UK

\subsection{6/gutjnl-2018-BSGAbstracts.74}

Introduction Adenoma miss rate during colonoscopy is considered as a key indicator of post colonoscopy cancer. One of the main reason for missed adenoma is poor visualisation of proximal folds and flexures during standard colonoscopy. Disposable distal attachments such as cap and Endocuff showed promising results to visualise proximal aspects of folds and to improve adenoma detection. There are no comparative randomised controlled trials of those two devices in the literature to date.

Aim Primary aim of this study was to compare adenoma miss rate between Endocuff assisted colonoscopy (EAC) and Cap assisted colonoscopy (CAC).

Methods All Patients referred for a colonoscopy via symptomatic services were invited to participate in the study. This is a randomised, single centre, tandem colonoscopy trial performed by the same endoscopist, on the same day first with Endocuff Vision followed by cap or vice-versa. All procedures were performed by Gastroenterology fellows who had performed more than 1500 colonoscopies each.

Results A total of 154 patients were recruited. 78 of them had CAC as their first procedure. The mean age of participant was 61 years (male: female was 1:1). Polyp miss rate was significantly lower in EAC (8.4\%) when compared with CAC $(26.1 \%, \mathrm{p}<0.001)$. Adenoma miss rate showed similar trend (EAC vs CAC, 6\%, vs $19 \%, \mathrm{p}=0.002$ ). Miss rate for diminutive adenomas where significantly lower in EAC group $(1.8 \%$ vs $19.6 \%, \mathrm{p}<0.001)$, however, there was no significant differences in the miss rates for small $(3.7 \%$ vs $2.9 \%, \mathrm{p}=0.69)$ or adenomas larger than $10 \mathrm{~mm}(2.6 \%$ vs $1.6 \%, \mathrm{p}=0.98)$. There was no significant difference in cancer, advanced adenoma or serrated polyps miss rate. The mean number adenoma per procedure was significantly higher in EAC group when compared to CAC (1.5 vs $0.8, \mathrm{p}<0.001)$.

Caecal intubation time were significantly shorter in the EAC group than CAC group (median 6 vs 7 mins, $p=0.01$ ) Conversely, withdrawal time (median 10 vs 8 mins, $\mathrm{p}=0.01$ ) was significantly longer in the EAC group. This difference could be due to more polyps were detected and resected in EAC group. While introducing the colonoscope via anus the discomfort score was higher in EAC $(55 \%$ vs $10 \%, \mathrm{p}<0.001)$. However, majority of them reported only a mild discomfort

During the first procedure, Endocuff was removed in 5/76 (6.6\%) patients' due to difficulties to pass the Endocuff through a fixed or narrowed sigmoid colon. There were no difficulties to negotiate the sigmoid colon when cap was used. There were no other colonoscopy related complications reported during the procedure.

Conclusions Our randomised, back to back study demonstrates that Endocuff assisted colonoscopy has significantly lower adenoma miss rate than cap assisted colonoscopy. Use of Endocuff appear to improve efficiency of the colonoscopy. 